\title{
Alveolar macrophages and CC chemokines are increased in children with cystic fibrosis
}

\author{
S. Brennan*,\#, P.D. Sly*,\#, C.L. Gangell*,\#, N. Sturges*,\#, K. Winfield*,\#, \\ M. Wikstrom*,\#, S. Gard*,* and J.W. Upham ${ }^{\star *, *+}$ on behalf of AREST CF ${ }^{\#, \S, f}$
}

ABSTRACT: Airway inflammation is an important component of cystic fibrosis (CF) lung disease. We sought to determine whether alveolar macrophages were involved in early CF lung disease.

Children with CF (median age $3.1 \mathrm{yrs}$ ) participated in a surveillance programme that included annual bronchoalveolar lavage (BAL). Control samples were obtained from non-CF children (median age 3.1 yrs; $\mathbf{n = 2 4 )}$ investigated for persistent respiratory symptoms.

Pulmonary infection was detected in $31 \%$ (16 out of 51 ) and $38 \%$ (nine out of 24) of children from the CF and non-CF groups, respectively. Alveolar macrophages in BAL were increased in CF compared with non-CF in the absence of infection $\left(223 \times 10^{3}\right.$ versus $\left.85 \times 10^{3} \mathrm{cells} \cdot \mathrm{mL}^{-1} ; p=0.001\right)$ and were associated with elevations in the CC chemokines (macrophage inflammatory protein (MIP)-3 $\alpha$ (chemokine (C-C motif) ligand (CCL)20; 355.8 versus $46.0 \mathrm{pg} \cdot \mathrm{mL}^{-1} ; \mathrm{p}<0.001$ ), monocyte chemotactic protein-1 (CCL2; 263.5 versus $\left.25.3 \mathrm{pg} \cdot \mathrm{mL}^{-1} ; \mathrm{p}<0.001\right)$, MIP-1 $\alpha$ (CCL3; 38.2 versus $\left.4.9 \mathrm{pg} \cdot \mathrm{mL}^{-1} ; \mathrm{p}<0.001\right)$ and MIP-1 $\left(\mathrm{CCL} 4 ; 326.6\right.$ versus $\left.27.5 \mathrm{pg} \cdot \mathrm{mL}^{-1} ; \mathrm{p}<0.001\right)$ ). Total cell counts and neutrophil numbers increased in the presence of infection; however, there was no additional effect of CF.

Alveolar macrophages and CC chemokines are elevated in the lungs in young children with CF even in the absence of pulmonary infection. Longitudinal studies are required to determine the clinical relevance of these findings.

KEYWORDS: CC chemokines, children, cystic fibrosis, macrophages

C ontroversy still exists over whether airway inflammation in young children with cystic fibrosis (CF) is a primary event or whether it arises only in response to pulmonary infection. Airway inflammation has been identified in young children with CF even in the absence of clinical or microbiological evidence of current infection [1-4], and has been associated with impaired lung function [3]. Understanding the mechanisms involved may result in more appropriately targeted therapy.

Previous attempts to understand the pathogenesis of airway inflammation in CF have focused on the role of neutrophils and the major neutrophil chemoattractant interleukin (IL)-8. Less attention has been paid to other leukocytes, such as macrophages and antigen-presenting cells, that are likely to initiate and regulate immune responses to pulmonary pathogens. Previous studies have investigated macrophages and dendritic cells in adults with more severe disease and chronic infections [5-9]. HubEAU et al. [10] reported an increased presence of alveolar macrophages in the lungs of late gestational fetuses with CF compared with non-CF fetuses. In addition, BRUSCIA et al. [11] reported that macrophages from CF transmembrane conductance regulator (CFTR) knockout mice show exaggerated inflammatory cytokine responses to bacterial lipopolysaccharide; in some cases these increases were dose dependent with intermediate changes present in heterozygotes. These data suggest that dysregulation of macrophage recruitment and/or function may be intrinsic to CF prior to the development of pulmonary infection and may be driven in some manner by CFTR dysfunction.

Monocytes, macrophages and dendritic cells are recruited to the lung by CC chemokines, especially monocyte chemotactic protein (MCP)-1 (CC chemokine ligand (CCL)2), macrophage inflammatory protein (MIP)-1 $\alpha$ (CCL3), MIP-1 $\beta$ (CCL4) and MIP-3 $\alpha$ (CCL20). These chemokines have received limited attention in $\mathrm{CF}$, with only isolated studies on the roles of MIP-3 $\alpha$ (CCL20)
AFFILIATIONS

*Telethon Institute for Child Health Research,

${ }^{*}$ Centre for Child Health Research, University of Western Australia, "Dept of Respiratory Medicine, Princess Margaret Hospital for Children, Perth,

+School of Medicine, The University of Queensland, Brisbane,

${ }^{\S}$ Murdoch Children's Research Institute, Melbourne, Australia. ${ }^{f}$ For details of the members of AREST CF, please see the Acknowledgements section.

CORRESPONDENCE

P.D. Sly

Division of Clinical Sciences, Telethon Institute for Child Health Research

P.0. Box 855

West Perth

WA 6872

Australia

E-mail: peters@ichr.uwa.edu.au

Received:

Nov 242008

Accepted after revision:

April 062009

First published online:

April 222009 
[12], RANTES (CCL5) [13, 14] and growth-regulated oncogene- $\alpha$ (CXC chemokine ligand 1) [15]. Animal studies have also demonstrated changes associated with CFTR dysfunction for MCP-1 (CCL2) and MIP-1 $\alpha$ (CCL3) [11].

The aims of the current study were to document the levels of CC chemokines (MCP-1, MIP- $1 \alpha$, MIP-1 $\beta$, MIP- $3 \alpha$ and RANTES) in the bronchoalveolar lavage fluid (BALF) of young children ( $<6$ yrs of age) with $\mathrm{CF}$ and to relate this to the numbers of macrophages in the lung. We hypothesised that children with CF would have higher levels of MCP-1, MIP-1 $\alpha$, MIP-1 $\beta$ and MIP-3 $\alpha$ in bronchoalveolar lavage (BAL) than children without $\mathrm{CF}$ but with persistent respiratory symptoms, and that these would be associated with increased numbers of alveolar macrophages in the lung.

\section{METHODS}

See online supplementary data for expanded methodology.

\section{Subject recruitment}

A total of 51 preschool-aged, nonexpectorating children with $\mathrm{CF}$ involved in the annual microbial surveillance programme conducted at Princess Margaret Hospital for Children, Perth, Australia, were assessed when clinically stable, defined as having no current clinical evidence of viral or bacterial infection or exacerbation of lung disease. 24 similarly aged children without $\mathrm{CF}$ who were undergoing investigation for persistent respiratory symptoms, predominantly recurrent or persistent moist cough, were also included (non-CF). The diagnosis of CF was confirmed by sweat test [16], following detection by either newborn screening or by clinical presentation [3]. The project was approved by the institutional ethics committee and parents gave consent for samples to be used.

\section{BAL processing}

Bronchoscopy and BAL was conducted under general anaesthesia according to the recommendations of the European Respiratory Society Task Force on BAL in children [17]. BALF was processed for detection of bacteria, fungi, viruses and inflammation using standard methodology as described elsewhere $[3,18,19]$. BAL was well tolerated in all subjects.

IL-8 was measured using a commercial ELISA (Biosource, Mount Waverley, Victoria, Australia; detection limit $20 \mathrm{pg} \cdot \mathrm{mL}^{-1}$ ). Neutrophil elastase activity was measured by a modified enzymatic assay [20] using $N$-succinyl Ala-Ala-Pro-Val as the specific substrate; the limit of the assay was $0.2 \mu \mathrm{g} \cdot \mathrm{mL}^{-1}$. IL- 6 was measured using a cytometric bead array (BD, North Ryde, New South Wales, Australia); the limit of the assay was $20 \mathrm{pg} \cdot \mathrm{mL}^{-1}$. Chemokines (MCP-1 (CCL2), MIP-1 $\alpha$ (CCL3), MIP-1 $\beta$ (CCL4) and RANTES (CCL5)) were chosen as they represented known chemotactic factors for macrophages and monocytes and/or have been investigated previously in older subjects with CF. They were measured by flow cytometry using a cytometric bead array (BD Biosciences, San Diego, CA, USA) with detection limits of $20 \mathrm{pg} \cdot \mathrm{mL}^{-1}$. MIP-3 $\alpha$ was measured by ELISA using a commercially available kit (R\&D Systems, Minneapolis, MN, USA; detection limit $\left.7.8 \mathrm{pg} \cdot \mathrm{mL}^{-1}\right)$. Samples below the lower limit of detection were arbitrarily assigned a value of half the lower limit of detection, in order to minimise the difficulties associated with statistical analysis of zero values. All soluble mediators were measured in duplicate, and were diluted to suit the linear portion of the curves, with inter- and intra-assay variation of $<5 \%$.

\section{Statistics}

Correlations between nonparametric variables were assessed using Spearman rank order correlation or the Pearson product moment test for parametric data. Differences between groups were assessed by unpaired t-test or by Wilcoxon rank test for nonparametric data. ANOVA on Ranks and ANOVA were used for comparisons between populations, and for comparisons of infection status within $\mathrm{CF}$ and non-CF groups for nonparametric and parametric data, respectively; adjustments for multiple comparisons were made using Dunn's method. Values below the limit of detection for assays were arbitrarily assigned a value one-half of the lowest level of detection for statistical purposes. Significant infection was defined as $>10^{4}$ colony forming units $(\mathrm{CFU}) \cdot \mathrm{mL}^{-1}$, while a density of $10^{2}-10^{4} \mathrm{CFU} \cdot \mathrm{mL}^{-1}$ was recorded as "isolated colonies". Statistical significance was achieved with a p-value of $<0.05$. Except where indicated, group data are presented as median, with interquartile range in parentheses. Analyses were conducted using Sigmastat (Systat Software Inc., Richmond, CA, USA).

\section{RESULTS \\ Study population}

A total of 51 children with CF (22 males, 29 females) with a median age of $3.07 \mathrm{yrs}$ (interquartile range (IQR) 1.10-4.04) were studied. The most common CF genotype was Phe508del, with $57 \%$ having two copies and 37\% having one copy. Only two children did not have this mutation. 24 children who did not have CF (nine females, 15 males), with a median age of $3.10 \mathrm{yrs}$ (IQR 1.79-4.91 yrs) and undergoing clinically indicated bronchoscopy and BAL, were included as a non-CF control group. The most common reason for BAL was investigation of chronic lower respiratory symptoms (83\%), with the remaining children investigated for structural airway disease. BAL return averaged $32.6 \%$ in the CF group and $52.6 \%$ in the non-CF group (see online supplementary data for further details).

\section{Pulmonary inflammation and infection}

Pulmonary infection was detectable in 31\% (16 out of 51) and in $38 \%$ (nine out of 24 ) of CF and non-CF groups, respectively. The most common organisms cultured in the CF group were Pseudomonas aeruginosa $(\mathrm{n}=6)$, Staphylococcus aureus $(\mathrm{n}=5)$ and Haemophilus influenzae $(\mathrm{n}=2)$, with a variety of other organisms seen. Of the CF group, $44 \%$ were taking oral antibiotics at the time of the BAL. The most common organisms cultured in the non-CF group were $H$. influenzae $(n=4)$ and Streptococcus pneumoniae $(n=2)$, with a variety of other organisms seen in individual children. Of the non-CF group, 74\% were taking oral antibiotics at the time of the BAL. As a group, children with CF had a significantly higher inflammatory burden, with higher total cell counts (median IQR) $\left(311 \times 10^{3}\left(203 \times 10^{3}-544 \times 10^{3}\right)\right.$ cells $\cdot \mathrm{mL}^{-1}$ versus $143 \times 10^{3}\left(114 \times 10^{3}-378 \times 10^{3}\right)$ cells $\left.\cdot \mathrm{mL}^{-1} ; \mathrm{p}=0.019\right)$, and a higher number of macrophages $\left(237 \times 10^{3}\left(149 \times 10^{3}-301 \times\right.\right.$ $\left.10^{3}\right)$ cells $\cdot \mathrm{mL}^{-1}$ versus $85 \times 10^{3}\left(49 \times 10^{3}-166 \times 10^{3}\right)$ cells $\cdot \mathrm{mL}^{-1}$; $\mathrm{p}=0.001)$, but not more neutrophils $(\mathrm{p}=0.53)$ than the non-CF group. A significant correlation was seen between the numbers of macrophages and the number of neutrophils in the BAL in both 
children with CF (fig. E1, online supplementary data) and those without CF (fig. E2, online supplementary data). In both groups, children with respiratory infections had higher cell counts (table 1). Children with CF also had higher levels of IL-8 in BAL $\left(0.22(0.1-0.65) \mathrm{ng} \cdot \mathrm{mL}^{-1}\right.$ versus $0.11 \quad(0.04-0.29) \mathrm{ng} \cdot \mathrm{mL}^{-1}$; $\mathrm{p}<0.001$; fig. E4, online supplementary data). Children with infections had significantly higher total cell counts $(p=0.001)$ and number of neutrophils ( $\mathrm{p}=0.001)$; however, there was no additional significant effect of disease (i.e. CF versus non-CF). Infected children with CF had the highest BAL neutrophil counts (table 1).

Higher levels of CC chemokines were found in the BALF of children with CF (fig. 1a-e; fig. E3a-e online supplementary data), including MIP-3 $\alpha$ (CCL20; $\mathrm{p}=0.002)$, MCP-1 (CCL2; $\mathrm{p}<0.001), \quad \mathrm{MIP}-1 \alpha \quad(\mathrm{CCL} 3 ; \mathrm{p}<0.001)$ and MIP-1 $\beta \quad(C C L 4 ;$ $p<0.001)$. There was no significant difference in RANTES (CCL5) levels between groups (fig. 1e). To account for the possibility that children defined as uninfected may have occult pulmonary infection, we conducted a separate analysis excluding children with any bacteria detected, i.e. "mixed oral flora" or "isolated colonies". 12 children with CF who had available chemokine data had absolutely no growth in BAL and had elevated BAL macrophages and CC chemokines when compared with non-CF, who cultured only scant bacteria or mixed oral flora (table E1, online supplementary data). The clinical features of both the CF and non-CF groups related to infection status are shown in table E2 (online supplementary data). Restricting the analysis to children aged $<18$ months, i.e. children with the lowest likelihood of having pulmonary infection, confirmed elevated BAL macrophages and CC chemokines in the CF group (table E3, online supplementary data).

There was a significant correlation between the number of macrophages in the BAL and MCP-1 (CCL2) in both CF and non-CF groups (fig. 2). There were also significant positive relationships between MIP-1 $\alpha$ (CCL3) and MIP-1 $\beta$ (CCL4) in BAL, and the number of macrophages in the BALF of children with CF (table E4, online supplementary data). These relationships were also seen in the non-CF group (data not shown). In contrast, IL-8, RANTES (CCL5) and MIP-3 $\alpha$ (CCL20) in BAL were not significantly associated with the number of macrophages in BAL in children with CF (table E3, online supplementary material). IL-8 was detected in the majority of children from both groups; in $68.6 \%$ of children with CF and $68.2 \%$ of those without $(p=1.0)$, with no difference in the levels between the groups $(p=0.15)$. BAL levels of IL- 8 were higher in the presence of pulmonary infection within the $C F$ group $(p=0.041)$ but not within the non-CF group (fig. $E 4$, online supplementary data).

Levels of IL-6, a cytokine known to regulate CC chemokine expression, in BAL correlated significantly with all CC chemokines: MCP-1 (CCL2; $\mathrm{r}=0.55, \mathrm{p}<0.0001), \mathrm{MIP}-1 \alpha$ (CCL3; $\mathrm{r}=0.55, \mathrm{p}<0.0001), \mathrm{MIP}-1 \beta$ (CCL4; $\mathrm{r}=0.56, \mathrm{p}<0.0001)$, MIP-3 $\alpha$ (CCL20; $\mathrm{r}=0.61, \mathrm{p}<0.0001)$ and RANTES (CCL5; $\mathrm{r}=0.38, \mathrm{p}=0.006$ ) (table E3, online supplementary material). More children with CF had detectable levels of IL-6 in their BAL $(27(52.9 \%)$ out of 51 versus four $(17.4 \%)$ out of 23 non-CF controls, $\mathrm{p}=0.005)$ and group mean levels were higher $(p=0.026)$. However, the influence of current infection on IL-6 levels failed to reach statistical significance in either group $(\mathrm{CF} p=0.057$; non-CF $\mathrm{p}=0.073$ ) (fig. E5, online supplementary data).

\section{DISCUSSION}

The results of the present study demonstrate that large numbers of macrophages and elevated levels of chemokines of the CC family, i.e. MCP-1 (CCL2), MIP-1 $\alpha$ (CCL3), MIP-1 $\beta$ (CCL4) and MIP-3 $\alpha$ (CCL20) are present in the lungs of young children with $\mathrm{CF}$ who have mild or no clinically apparent lung disease. The levels were not significantly increased further in

TABLE 1 Inflammatory mediators in bronchoalveolar lavage fluid (BALF) of cystic fibrosis (CF) and non-CF groups

\begin{tabular}{|c|c|c|c|c|}
\hline \multirow[t]{2}{*}{ Characteristics } & \multicolumn{2}{|c|}{ Non-CF } & \multicolumn{2}{|c|}{$\mathrm{CF}$} \\
\hline & Uninfected & Infected & Uninfected & Infected \\
\hline Subjects n & 15 & 9 & 35 & 16 \\
\hline TCC $\times 10^{3} \cdot \mathrm{mL}^{-1}$ BALF & $121(104-291)$ & $548(169-1037)^{\star}$ & $278(163-424)^{\#}$ & $500(311-945)^{*}$ \\
\hline Macrophages $\times 10^{3} \cdot \mathrm{mL}^{-1}$ & $85(50-134)$ & $112(39-344)$ & $223(148-283)^{\#}$ & 284 (198-383) \\
\hline Macrophages \% & $63.3(45.7-77.9)$ & $32.7(32.0-46.3)$ & $60.7(69.0-91.2)^{\#}$ & $61.3(56.5-75.0)^{*}$ \\
\hline Neutrophils \% TCC & $29.3(13.5-37.0)$ & $38.7(18.7-64.3)$ & $18.8(7.3-30.7)$ & $37.7(12.9-66.0)^{\star}$ \\
\hline $\mathrm{IL}-8 \mathrm{ng} \cdot \mathrm{mL}^{-1}$ & $0.10(0.05-0.21)$ & $0.12(0.09-0.39)$ & $0.19(0.09-0.35)^{\#}$ & $0.52(0.15-3.61)^{*, \#}$ \\
\hline Detectable NE & $1(7)$ & $2(11)$ & $6(17)$ & $8(50)^{*}$ \\
\hline $\mathrm{NE} \mu \mathrm{g} \cdot \mathrm{mL}^{-1}$ & $0.10(0.10-0.10)$ & $0.10(0.10-0.10)$ & $0.10(0.10-0.10)$ & $0.62(0.10-2.00)^{*}$ \\
\hline $\mathrm{MCP}-1 \mathrm{pg} \cdot \mathrm{mL}^{-1}$ & $24.75(16.30-29.40)$ & $49.30(15.07-131.40)$ & $177.35(51.3-327.1)^{\#}$ & $242.2(108.85-347.64)^{\#}$ \\
\hline MIP-1 $\alpha \mathrm{pg} \cdot \mathrm{mL}^{-1}$ & $4.3(3.05-6.0)$ & $12.2(4.42-68.87)$ & $24.2(11.5-45.3)^{\#}$ & $37.3(14.5-46.98)$ \\
\hline
\end{tabular}

Data are presented as median (interquartile range) or $\mathrm{n}(\%)$, unless otherwise indicated. TCC: total cell count; IL: interleukin; NE: neutrophil elastase; MCP: monocyte chemotactic protein; MIP: macrophage inflammatory protein. *: $p<0.05$ between uninfected and infected subjects; ${ }^{*}: p<0.05$ between $C F$ and non-CF subjects. 

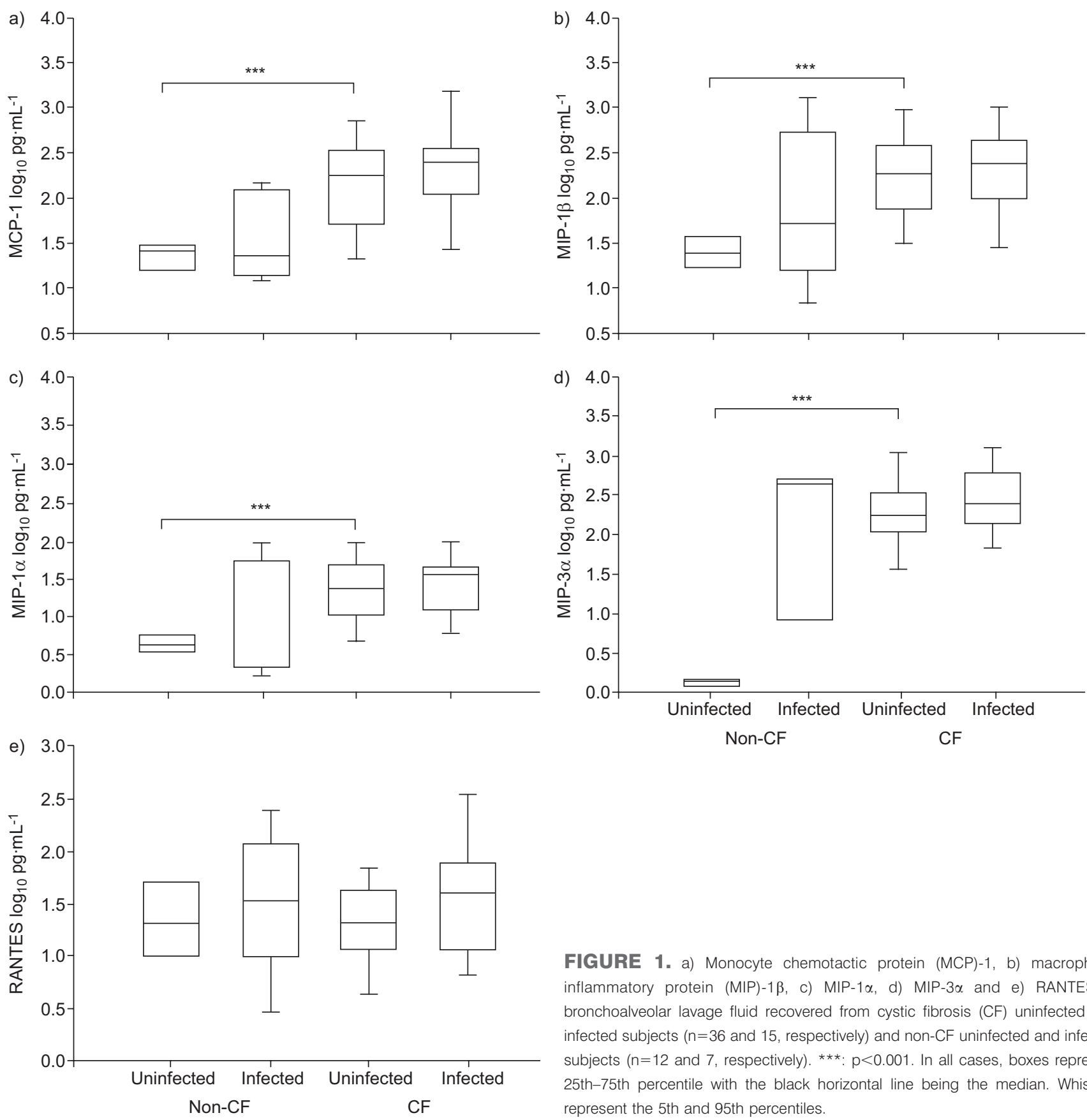

FIGURE 1. a) Monocyte chemotactic protein (MCP)-1, b) macrophage inflammatory protein (MIP)-1 $\beta$, c) MIP- $1 \alpha$, d) MIP- $3 \alpha$ and e) RANTES in bronchoalveolar lavage fluid recovered from cystic fibrosis (CF) uninfected and infected subjects ( $\mathrm{n}=36$ and 15, respectively) and non-CF uninfected and infected subjects ( $n=12$ and 7 , respectively). ${ }^{* *}: p<0.001$. In all cases, boxes represent 25th-75th percentile with the black horizontal line being the median. Whiskers represent the 5th and 95th percentiles.

the presence of pulmonary infection in the CF group, suggesting that the genetic defect in CF is likely to be at least partly responsible. In the presence of pulmonary infection, the total cell counts and number of neutrophils increased in the lungs of children in both the CF and non-CF groups.

CF lung disease is characterised by intense inflammation, repeated bacterial infections and progressive lung destruction, and there is clearly a need to understand the mechanisms involved early in the disease if new forms of early intervention are to be developed. The inflammatory response to respiratory pathogens is thought to be exaggerated in both intensity and duration. Much research on the pathogenesis of CF lung

disease has appropriately been directed towards neutrophil function, given that neutrophils are the predominant cell type identified in the CF lung during chronic disease. The role of other leukocytes in airway inflammation in CF has received less attention, although the results of the current study suggest that the role of macrophages should not be ignored in early $\mathrm{CF}$ lung disease. Lung macrophages play an important role in host defence and regulation of inflammation by interactions with inflammatory cells, including an important interaction with neutrophils. Macrophages contribute to the innate response by release of cytokines and mediators, such as IL- 8 and tumour necrosis factor (TNF)- $\alpha$, and by facilitating neutrophil influx and efflux [21]. 


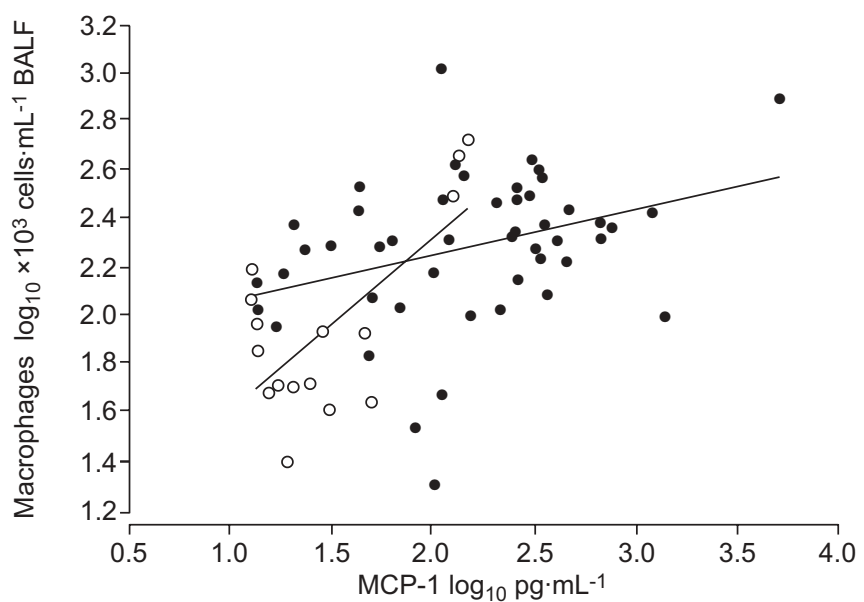

FIGURE 2. Relationship between the number of macrophages per millilitre of bronchoalveolar lavage fluid (BALF) retrieved from cystic fibrosis (CF) subjects ( and non-CF (O) subjects and monocyte chemotactic protein (MCP)-1 levels in BALF. Non-CF: slope $=0.699$, intercept $=0.923, r=0.677 ; \quad C F$ : slope $=0.182$, intercept $=1.884, r=0.328$.

In the present study, we noted that children with CF in the "infected" group had an increase in the absolute number of macrophages compared with the non-CF "infected" group, and that the number of macrophages was correlated with the CC chemokines measured. Interestingly, we simultaneously noted a relatively larger decrease in the percentages of macrophages in the BAL that did not reach significance associated with infection status. These differences (i.e. increases in absolute numbers compared with decreases in percentages) could be a reflection of the overall inflammatory influx seen in CF compared with non-CF subjects, with relatively larger numbers of neutrophils migrating into the lung. This change in cell profile with infection may impact on the inflammatory environment. With relatively fewer macrophages present during a pulmonary infection, there may be a relatively diminished ability to phagocytose the increasing population of dying neutrophils. Cell necrosis and the release of proteolytic enzymes are more likely under these circumstances and may contribute to the cycle of chronic inflammation that ensues. However, the relative change in the percentage of macrophages present in $\mathrm{CF}$ versus non-CF children with the influence of infection in this study would need to be confirmed by larger numbers and serial changes within individuals as they go from an uninfected to an infected state. However, specific studies targeting children before and during pulmonary infection would be problematic; these changes could be observed opportunistically in children with $\mathrm{CF}$ in surveillance programmes as they progress through their illness.

Animal models have provided much important information about chemokines and the role of CFTR to date. BRUSCIA et al. [11] demonstrated that stimulated macrophages in CFTR knockout, heterozygote and wild-type mice resulted in a variation of cytokine and chemokine production (CCL2) that was related to genotype. A similar relationship has been reported in patients with $\mathrm{CF}$, with higher circulating levels of CCL2 and IL-8 in those with genotypes associated with more severe phenotypes [13]. Other animal studies have shown chemokines to have a central role in the development of allergic aspergillosis, a complication seen in CF [22]. In those models, CCL2 has mixed effects depending on the disease progression; other CC chemokine receptors (CCR2, CCR4 and CCR5) also contribute to disease aspects including airway hyperresponsiveness, phagocytosis and bacterial killing. The role of CC chemokines has not been previously examined in early CF lung disease in a well-characterised, clinically stable population. However, studies in older patients lend support to the findings from the present study. Previous studies have reported that MCP-1 (CCL2) [23], but not RANTES (CCL5), is elevated in older patients with more advanced lung disease $[13,24]$. MIP-3 $\alpha$ (CCL20) is elevated in primary CF epithelial cell cultures [12]. MCP-1 (CCL2) and MIP-3 $\alpha$ (CCL20) have both been reported to be elevated in BALF in subjects with CF $[12,25]$ and in induced sputum and blood (CCL2) [23] compared with controls. MIP-1 $\alpha$ (CCL3) has also been identified as being elevated in the tears of subjects with $\mathrm{CF}$ compared with healthy controls [26].

CC chemokines play an important role in both recruitment of macrophages to the lung and in the resolution of inflammation $[25,27-29]$. CC chemokines are regulated by cytokines, such as IL-1 $\beta$, TNF- $\alpha$ and IL-6, and this direct relationship with IL-6 was evident in the present study. MCP-1 (CCL2), MIP-1 $\alpha$ and $-1 \beta$ (CCL3 and CCL4, respectively) and MIP-3 $\alpha$ (CCL20) are known chemoattractants for activated macrophages, which are recruited to the lung upon exposure to $\mathrm{C} 5 \mathrm{a}$ or bacterial proteins. These cells tend to have a different profile from the resident lung macrophages, being smaller in size [30], producing different inflammatory cytokines and potentially having impaired phagocytosis [31]; they may thus be less able to assist in resolution of inflammation. Unfortunately, during our study, we were unable to isolate monocytes from BAL to investigate this further. However, a recent study by RAO et al. [23] demonstrated increased CCL2 in blood and sputum in older patients with CF compared with controls, with no difference in expression of the receptor CCR2 on monocytes. Macrophages play a vital role in resolution of inflammation, especially in the ingestion and clearance of apoptotic neutrophils $[27,28]$, and CC chemokines are intimately involved in the co-ordination of that role. Furthermore, there is mounting evidence that in $\mathrm{CF}$, macrophages may have impaired function in their ability to phagocytose and to clear apoptotic neutrophils $[8,9,32]$ and this may be due to CFTR effects, with possible mechanisms being defects in intracellular chloride levels and the inability to adequately acidify lysosomes [32]. Taken together, these data suggest the hypothesis that, from early infancy, there is a high recruitment of phenotypically "small" monocytes to the airways by CC chemokines. These monocytes may not be competent to phagocytose, resulting in an ineffective phagocytic response following stimulation leading to the accumulation of necrotic neutrophils. Unfortunately, the data from the present study do not shed any light on this hypothesis.

In the current study, the most striking finding was that apparently uninfected children with CF had significantly elevated levels of the CC chemokines MCP-1 (CCL2), MIP-1 $\alpha$ (CCL3), MIP-1 $\beta$ (CCL4) and MIP-3 $\alpha$ (CCL20) in BALF compared with children with non-CF lung disease. These 
findings were seen both in the CF group as a whole, and also in the youngest children aged $<18$ months. It is unlikely that these findings can be attributed to technical factors, as a standardised method was employed for collecting BAL from all children [17] and the samples were handled in an identical manner in the microbiology laboratory. Misclassification of infection status is one possible explanation for the apparent similarity in chemokine levels between infected and uninfected children with CF. Some of the children classified as "uninfected" may have had low numbers of pathogens in their lungs that were below the threshold for detection by standard culture as we used a very conservative definition of infection (i.e. $>10^{4} \mathrm{CFU} \cdot \mathrm{mL}^{-1}$ ). However, there was no statistical association between isolation of low numbers of pathogens $\left(10^{2}-10^{4} \mathrm{CFU} \cdot \mathrm{mL}^{-1}\right)$ and chemokine levels in BAL (data not shown). We cannot exclude the possibility that chemokine production and macrophage influx in $\mathrm{CF}$ are driven by focal infection in a region of the lung that was not sampled by BAL, and this issue would only be addressed by sampling a greater number of lobes in future studies. However, there are practical and ethical limitations to such an approach.

Some of the children with CF may have had a recent, unrecognised infection from which they had recovered, and this might explain the chemokine and macrophage data. However, all the BAL samples were obtained from CF children when they were clinically stable and none had viral infections detected in BAL. In addition, there were no associations between respiratory symptom scores at the time of the procedure and BAL macrophage counts or chemokine levels (data not shown). Likewise, neutrophil numbers were not different between uninfected $\mathrm{CF}$ and uninfected non-CF groups (table 1), and were relatively low, suggesting that neutrophilic inflammation does not account for the difference in chemokine levels found. Furthermore, an assessment of chemokine levels versus bacterial density failed to show any correlation with CC chemokines measured. However, with all of these considerations, it nonetheless remains possible that elevated CC chemokine production and macrophage recruitment to the lung may have been a consequence of recent undetected or current subclinical infection. We do have to acknowledge that our control group may not have been the most ideal. Healthy children undergoing elective surgery for nonrespiratory reason may have provided a more appropriate comparison. However, performing BAL in these children is not a trivial procedure, and we did not consider it to be justifiable. Our group of non-CF disease controls did provide the ability to look at both currently infected and currently uninfected children for comparison with our CF group.

A variety of noninfective factors could also contribute to inflammation in CF, including impaired neutrophil clearance [9] and oxidative stress [33]. Finally, it is also possible that dysregulation of chemokine production and macrophage recruitment can occur independently in children with CF and may be an early event in the initiation of lung disease. This would be consistent with the finding that lung macrophages are increased in the lungs of fetuses with CF [10] and in CFTR knockout mouse studies [11], suggesting that monocyte recruitment and function is an issue that is worthy of further investigation as a target for therapeutic intervention.
The levels of CC chemokines found in BALF in our CF population were considerably elevated (up to 10 times) above those of the disease controls and, given the significant correlations between several chemokines and numbers of macrophages (fig. 2) and neutrophils (table E4, online supplementary data) in BAL, we may speculate that these chemokines are inducing recruitment of various leukocyte populations from the blood to the lung.

There have been relatively few prospective bronchoscopic studies that examine early-stage lung disease in young children with CF who are clinically stable. Despite the difficulties of sampling this population, these studies are important in order to gain insight into the early stages of lung disease. The subjects in our study represent a unique cohort of children who have had annual assessment of BAL from early in infancy, are at a time of clinical stability and have minor inflammatory lung disease. Assessment is conducted on an annual basis for the purpose of microbiological surveillance, rather than during respiratory exacerbations, which is often the case in other studies reported in the literature. As this cohort is followed up over the coming years, it will be important to determine the relationship between macrophage accumulation in the lung, CC chemokine expression and the subsequent clinical course of these children.

\section{SUPPORT STATEMENT}

The Australian Cystic Fibrosis Research Trust (North Ryde, NSW, Australia), the Australian National Health and Medical Research Council (Canberra, ACT) and the Cystic Fibrosis Foundation (Bethesda, MD, USA) provided financial support for the present study.

\section{STATEMENT OF INTEREST}

None declared.

\section{ACKNOWLEDGEMENTS}

The authors would like to thank all subjects with CF and their families, A. Krishnan (Microbiology, Princess Margaret Hospital, Perth, Western Australia) for her technical assistance, S.M. Stick, A. Martin, T. Douglas, A. Schultz and the Dept of Respiratory Medicine at the Princess Margaret Hospital (Perth) for their assistance with sample collection.

The members of the Australian Respiratory Early Surveillance Team for Cystic Fibrosis (AREST CF) are: L. Berry, S. Brennan, C. Calogero, N. De Klerk, C.L. Gangell, L. Garratt, A. Kicic, I. Laing, L. Mott, G. Nolan, P.D. Sly, E. Sutanto (Division of Clinical Sciences, Telethon Institute for Child Health Research and Centre for Child Health Research, University of Western Australia, Perth); R. Robins-Browne, J. Carlin, R. Carzino, B. Skoric (Murdoch Children's Research Institute, Melbourne); E. Balding, T. Douglas, G.L. Hall, D. Mullane, C. Murray, S. Reddy, S.M. Stick (Dept of Respiratory Medicine, Princess Margaret Hospital for Children, Perth); and B. Linnane, J. Massie, N. Pillarisetti, S. Ranganathan, P.J. Robinson, C.F. Robertson (Dept of Respiratory Medicine, Royal Children's Hospital, Melbourne).

\section{REFERENCES}

1 Armstrong D, Grimwood K, Carzino R, et al. Lower respiratory infection and inflammation in infants with newly diagnosed cystic fibrosis. BMJ 1995; 310: 1571-1572.

2 Balough K, McCubbin M, Weinberger M, et al. The relationship between infection and inflammation in the early stages of lung disease from cystic fibrosis. Pediatr Pulmonol 1995; 20: 63-70. 
3 Brennan S, Hall GL, Horak F, et al. Correlation of forced oscillation technique in preschool children with cystic fibrosis with pulmonary inflammation. Thorax 2005; 60: 159-163.

4 Khan TZ, Wagener JS, Bost T, et al. Early pulmonary inflammation in infants with cystic fibrosis. Am J Respir Crit Care Med 1995; 151: 1075-1082.

5 Kerby GS, Cottin V, Accurso FJ, et al. Impairment of macrophage survival by $\mathrm{NaCl}$ : implications for early pulmonary inflammation in cystic fibrosis. Am J Physiol Lung Cell Mol Physiol 2002; 283: L188-L197.

6 Knight RA, Kollnberger S, Madden B, et al. Defective antigen presentation by lavage cells from terminal patients with cystic fibrosis. Clin Exp Immunol 1997; 107: 542-547.

7 Roghanian A, Drost EM, MacNee W, et al. Inflammatory lung secretions inhibit dendritic cell maturation and function via neutrophil elastase. Am J Respir Crit Care Med 2006; 174: 1189-1198.

8 Vandivier RW, Fadok VA, Hoffmann PR, et al. Elastase-mediated phosphatidylserine receptor cleavage impairs apoptotic cell clearance in cystic fibrosis and bronchiectasis. J Clin Invest 2002; 109: 661-670.

9 Vandivier RW, Fadok VA, Ogden CA, et al. Impaired clearance of apoptotic cells from cystic fibrosis airways. Chest 2002; 121: Suppl. 3,89 S.

10 Hubeau C, Puchelle E, Gaillard D. Distict patterns of immune cell populations in the lung of human fetuses with cystic fibrosis. J Allergy Clin Immunol 2001; 108: 524-529.

11 Bruscia E, Zhang P-X, Ferreira E, et al. Macrophages directly contribute to the exaggerated inflammatory response in $\mathrm{CFTR}^{-/}$ mice. Am J Respir Cell Mol Biol 2009; 40: 295-304.

12 Starner T, Barker C, Jia H, et al. CCL20 is an inducible product of human airway epithelia with innate immune properties. Am J Respir Cell Mol Biol 2003; 29: 627-633.

13 Augarten A, Paret G, Avneri I, et al. Systemic inflammatory mediators and cystic fibrosis genotype. Clin Exp Med 2004; 4: 99-102.

14 Koller DY, Nething I, Otto J, et al. Cytokine concentrations in sputum from patients with cystic fibrosis and their relation to eosinophil activity. Am J Respir Crit Care Med 1997; 155: 1050-1054.

15 Wyatt HA, Sampson AP, Balfour-Lynn IM, et al. Production of the potent neutrophil chemokine, growth-related protein $\alpha(\mathrm{GRO}-\alpha)$, is not elevated in cystic fibrosis children. Respir Med 2000; 94: 106-111.

16 Gibson LE, Cooke RE. A test for concentration of electrolytes in sweat in cystic fibrosis of the pancreas utilizing pilocarpine by iontophoresis. Pediatrics 1959; 23: 545.

17 de Blic J, Midulla F, Badbato A, et al. Bronchoalveolar lavage in children. Eur Respir J 2000; 15: 217-231.
18 Franklin P, Hall G, Moeller A, et al. Exhaled nitric oxide is not reduced in infants with cystic fibrosis. Eur Respir J 2006; 27: 350-353.

19 Moeller A, Horak F, Lane C, et al. Expression of inducible nitric oxide synthase gene in airway epithelial cells in young children with cystic fibrosis. Thorax 2006; 61: 514-520.

20 Delacourt C, Herigault S, Delclaux C, et al. Protection against acute lung injury by intravenous or intratracheal pretreatment with EPIHNE-4, a new potent neutrophil elastase inhibitor. Am J Respir Cell Mol Biol 2002; 26: 290-297.

21 Lambrecht B. Alveolar macrophage in the driver's seat. Immunity 2006; 24: 366-368.

22 Hartl D, Buckland K, Hogaboam C. Chemokines in allergic aspergillosis - from animal models to human lung diseases. Inflamm Allergy Drug Targets 2006; 5: 219-228.

23 Rao S, Wright AK, Montiero W, et al. Monocyte chemoattractant chemokines in cystic fibrosis. J Cyst Fibros 2009; 8: 97-103.

24 Hartl D, Latzin P, Zissel G, et al. Chemokines indicate allergic bronchopulmonary aspergillosis in patients with cystic fibrosis Am J Respir Crit Care Med 2006; 173: 1370-1376.

25 Hartl D, Griese M, Kappler M, et al. Pulmonary T(H)2 response in Pseudomonas aeruginosa-infected patients with cystic fibrosis. J Allergy Clin Immunol 2006; 117: 204-211.

26 Mrugacz M, Zelazowska B, Bakunowicz-Lazarczyk A, et al. Elevated tear fluid levels of MIP- $1 \alpha$ in patients with cystic fibrosis. J Interferon Cytokine Res 2007; 27: 491-495.

27 Amano H, Morimoto K, Senba M, et al. Essential contribution of monocyte chemoattractant protein-1/C-C chemokine ligand-2 to resolution and repair processes in acute bacterial pneumonia. J Immunol 2004; 172: 398-409.

28 Ariel A, Fredman G, Sun YP, et al. Apoptotic neutrophils and T cells sequester chemokines during immune response resolution through modulation of CCR5 expression. Nat Immunol 2006; 7: 1209-1216.

29 Marriott HM, Hellewell PG, Cross SS, et al. Decreased alveolar macrophage apoptosis is associated with increased pulmonary inflammation in a murine model of pneumococcal pneumonia. J Immunol 2006; 177: 6480-6488.

30 Rao S, Grigg J. New insights into pulmonary inflammation in cystic fibrosis. Arch Dis Child 2006; 91: 786-788.

31 Gordon S. The macrophage: past, present and future. Eur J Immunol 2007; 37: S9-S17.

32 Di A, Brown ME, Deriy LV, et al. CFTR regulates phagosome acidification in macrophages and alters bactericidal activity. Nat Cell Biol 2006; 8: 933-944.

33 Kettle AJ, Chan T, Osberg I, et al. Myeloperoxidase and protein oxidation in the airways of young children with cystic fibrosis. Am J Respir Crit Care Med 2004; 170: 1317-1323. 